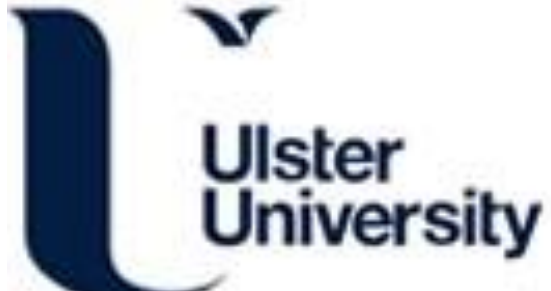

\section{Carbon nanotube growth activated by quantum-confined silicon nanocrystals}

Mariotti, D., Svrcek, V., Mathur, A., Dickinson, C., Matsubara, K., \& Kondo, M. (2013). Carbon nanotube growth activated by quantum-confined silicon nanocrystals. Journal of Physics D: Applied Physics, 46(12), 122001. http://stacks.iop.org/0022-3727/46/i=12/a=122001

Link to publication record in Ulster University Research Portal

Published in:

Journal of Physics D: Applied Physics

Publication Status:

Published (in print/issue): 01/01/2013

\section{Document Version}

Publisher's PDF, also known as Version of record

\section{General rights}

Copyright for the publications made accessible via Ulster University's Research Portal is retained by the author(s) and / or other copyright owners and it is a condition of accessing these publications that users recognise and abide by the legal requirements associated with these rights.

\section{Take down policy}

The Research Portal is Ulster University's institutional repository that provides access to Ulster's research outputs. Every effort has been made to ensure that content in the Research Portal does not infringe any person's rights, or applicable UK laws. If you discover content in the Research Portal that you believe breaches copyright or violates any law, please contact pure-support@ulster.ac.uk. 
Carbon nanotube growth activated by quantum-confined silicon nanocrystals

This article has been downloaded from IOPscience. Please scroll down to see the full text article.

2013 J. Phys. D: Appl. Phys. 46122001

(http://iopscience.iop.org/0022-3727/46/12/122001)

View the table of contents for this issue, or go to the journal homepage for more

Download details:

IP Address: 78.153.58.145

The article was downloaded on 19/02/2013 at 22:52

Please note that terms and conditions apply. 


\title{
Carbon nanotube growth activated by quantum-confined silicon nanocrystals
}

\author{
D Mariotti ${ }^{1}$, V Švrček ${ }^{2}$, A Mathur ${ }^{1}$, C Dickinson ${ }^{3}$, K Matsubara $^{2}$ and \\ M Kondo ${ }^{2}$ \\ ${ }^{1}$ NIBEC, University of Ulster, Shore Road, Newtownabbey, BT37 0QB, UK \\ ${ }^{2}$ Research Center for Photovoltaic Technologies, AIST, Tsukuba, 305-8568, Japan \\ ${ }^{3}$ Materials and Surface Science Institute, University of Limerick, Limerick, Ireland \\ E-mail: d.mariotti@ulster.ac.uk (D Mariotti)
}

Received 18 December 2012, in final form 28 January 2013

Published 18 February 2013

Online at stacks.iop.org/JPhysD/46/122001

\begin{abstract}
We report on the use of silicon nanocrystals (Si-ncs) to activate nucleation and growth of carbon nanotubes (CNTs) without using any metal catalyst. Si-ncs with different surface characteristics have been exposed to the same $\mathrm{CH}_{4}$ low-pressure plasma treatment producing quite different results. Specifically, Si-ncs prepared by laser ablation in water have contributed to the formation of micrometre-sized silicon spherical particles. On the other hand, Si-ncs prepared by electrochemical etching did not induce any specific growth while the third type of Si-ncs, prepared by electrochemical etching and treated by a laser fragmentation process, induced the growth of multi-walled CNTs. The different outcomes of the same plasma process are attributed to the diverse surface features presented by the Si-ncs.
\end{abstract}

S Online supplementary data available from stacks.iop.org/JPhysD/46/122001/mmedia

Silicon (Si) and carbon (C) are two of the most abundant elements in nature and play a crucial role in many of the current technologies; it is not by chance that these are also two of the most environmentally friendly elements. Both elements present a wide range of properties and characteristics that could possibly suit many of the future technological needs as they can provide a large selection of nanoscale structures with diverse physical and chemical characteristics [1,2].

For instance, while bulk $\mathrm{Si}$ is an indirect band-gap semiconductor with poor optical emission properties, when Si nanocrystals (Si-ncs) are synthesized with $5 \mathrm{~nm}$ diameter or less, the energy band gap opens up and novel optoelectronic properties appear [1]. As a result, the recombination processes (e.g. Auger) in Si-ncs can be tuned and the emission or absorption of photons occurs at different energies that depend on the nanocrystal diameter. Si-ncs with diameters below $10 \mathrm{~nm}$ are also significantly affected by the surface-to-volume ratio where the surface characteristics can determine the overall behaviour of the nanocrystal. Therefore, the combined effects of quantum confinement and surface characteristics can present favourable and unique properties for the application of Si-ncs in optoelectronic and photovoltaic (PV) devices [3].

Similarly, carbon nanomaterials exhibit interesting properties that could be very useful in many different application devices and have been intensively studied by the scientific community for the last couple of decades [2]. Carbon offers a large set of nanostructures that can potentially allow the design and fabrication of complex and functional nanoarchitectures to include spherical, monolayer or tubular complexes [4-6]. This is possible thanks to advanced and established technologies such as chemical vapour deposition, pyrolysis of hydrocarbons, plasma-assisted processes, etc [7]. Particularly, carbon nanotubes (CNTs) have played a central role in nanotechnology research for many years. Although CNT device integration has presented various challenges, there have been tremendous advances and their use in a wide range of applications is still very promising [8-12].

It is therefore very attractive to couple $\mathrm{Si}$ - and $\mathrm{C}$ nanostructures to form advanced nanoscale device components for a wide range of applications to include optoelectronics, 
PVs, energy storage, bio-imaging, drug delivery, etc [13-17]. One of the most attractive opportunities is for instance offered by $\mathrm{Si}$-ncs/CNT junctions that have so far received very limited attention mainly due to the difficulties in the synthesis process [13]. Another interesting combination is also offered by $\mathrm{Si}$-ncs/ $\mathrm{C}_{60}$ architectures which together offer advantageous characteristics for solar cell applications [18]. Si- and C-based nanostructures can offer the possibility of exploring new nanodevice architectures that may open the way to alternative and improved design approaches. In general, it is believed that optoelectronic, electronic and PV devices could be based on entirely different geometries that can considerably enhance the unique properties and the new physics originating from nanomaterials [19]. In order to achieve electronic coupling between $\mathrm{Si}$ - and $\mathrm{C}$-nanostructures and to produce functional nanoarchitectures, the synthesis processes have to be studied and optimized. It is interesting to note that in the same way surface/interface characteristics affect the nanostructure properties, the surface features can also significantly affect the outcomes of nanofabrication techniques that are used to produce functional devices.

Efforts have been made to combine quantum-confined semiconducting nanoparticles with one-dimensional nanostructures [20] or to combine different Si-based materials with CNTs [13-16]; however, no attempt has been reported to study CNT growth using quantum-confined Si-ncs catalysts. A Sinc/CNT nanostructure would represent an almost ideal fundamental opto-electronic device component where the CNT can serve as the acceptor to promote exciton dissociation and as an efficient charge transport channel for the flow of carriers to the collecting electrode.

Both CNTs (depending on chirality) and Si-ncs can act as the light harvesting component optimized for different spectral regions. At the same time, the same composite nanostructure can have a wide range of other applications. One of such applications is for instance energy storage [15]. Si-nc/CNT composites could be used for faster, lighter and longer-lasting Li-ion batteries due to the very large volumetric and gravimetric capacity of silicon with up to $3580 \mathrm{mAhg}^{-1}$ at room temperature [15]. This type of rechargeable battery could take advantage of CNT-connected Si-ncs; this $\mathrm{Si} / \mathrm{C}$ nanocomposite could contribute to faster charge/discharge cycles where the mechanical stability of silicon may be enhanced by the rigid CNT structure attached to the nanocrystals [21]. Based on this idea, experimental device batteries have been tested whereby silicon nanoparticles were grown on vertically aligned CNTs with very promising results [15].

Here, we report on the synthesis and integration of Si-ncs with carbon-based nanostructures such as CNTs. Different types of Si-ncs have been synthesized by different techniques and with different surface features. We demonstrate that the surface characteristics of the $\mathrm{Si}$-ncs determine the end result after exposure to the same $\mathrm{CH}_{4}$ low-pressure plasma treatment and that CNT growth is observed for only one type of Si-ncs. In the other cases different outcomes are produced and results will be also discussed [22]. Specifically, three different types of Si-ncs are considered here: $\mathrm{Si}$ ncs produced by laser ablation in water (LA-Si-ncs), Sincs produced by electrochemical etching (EE-Si-ncs) and electrochemical etched $\mathrm{Si}$-ncs that have been subsequently fragmented by laser processing in water (LF-Si-ncs). The three different types of Si-ncs present very different surface characteristics that determine the corresponding optoelectronic properties (see the online supporting information for more details http://stacks.iop.org/JPhysD/46/122001/mmedia) and have been exposed to the same microwave low-pressure plasma treatment $\left(\mathrm{Ar}+\mathrm{CH}_{4}\right)$. Figure 1 reports scanning electron microscopy (SEM) images of Si-ncs after plasma treatment for all three cases. After plasma treatment LA-Si-ncs formed crystalline micrometre-sized Si spheres (figure 1(a)). EESi-ncs did not show any particular morphological change (figure $1(b)$ ) and LF-Si-ncs have instead induced the growth of CNTs and other fibre-like structures (figure 1(c)).

The formation of spherical large particles shown in figure 1(a) was observed in our previous work with the same type of LA-Si-ncs [22]; on that occasion LA-Si-ncs were treated with acetylene plasma with very similar results. Our previous report provides detailed characterization of the spherical particles which also resulted in this case to be large single crystal spherical particles [22]. EE-Si-ncs did not produce any specific change after the same plasma processing, possibly with only amorphous carbon deposited on the sample. Significant changes were, however, seen in the sample with LF-Si-ncs. In particular, the sample has shown the formation of fibre-like structures with lengths exceeding $1 \mu \mathrm{m}$ (inset of figure $1(c)$ ) with diameter in the range of about $30 \mathrm{~nm}$.

A more detailed transmission electron microscopy (TEM) analysis has shown that the fibrous structures are for the most part represented by multi-walled CNTs (MWCNTs; figure 2). The spacing between the walls is $\sim 0.34 \mathrm{~nm}$ correlating with the $\left(\begin{array}{lll}0 & 0\end{array}\right) d$-spacing of graphite. High-resolution TEM images show occasional internal capping or bamboo structure which can be seen in figure $2(a)$ and is common in MWCNTs. Figure 2(b) also shows disruption of the CNTs walls which leads to a rather torturous nature of the CNTs. TEM analysis also reveals the presence of Si-ncs inside the tube (figure 3) where the presence of silicon at that location was confirmed by energy-dispersive x-ray spectroscopy (not shown here).

It is important to note that CNT growth has been achieved here with no transition metal catalysts. The limited number of single Si-ncs and the presence of large aggregates may have limited the growth yield and has determined the random and tortuous nature of the MWCNTs. Although we do not have at this stage sufficient evidence to comment on the role played by the LF-Si-ncs on the CNT growth, it is clear that Si-ncs have had an important effect and possibly have acted as catalyst particles for the CNT growth.

The mechanisms that allow CNT growth on non-metallic catalysts are not yet fully understood. There is some consensus that due to the generally higher melting temperature of non-metallic catalysts (e.g. $\mathrm{SiO}_{2}$ ), the vapour-liquidsolid mechanism cannot take place and vapour-solid-solid (VSS) or vapour-solid surface-solid (VSSS) mechanisms have been proposed $[12,14,23]$. Essentially there are two 
IOP FTC

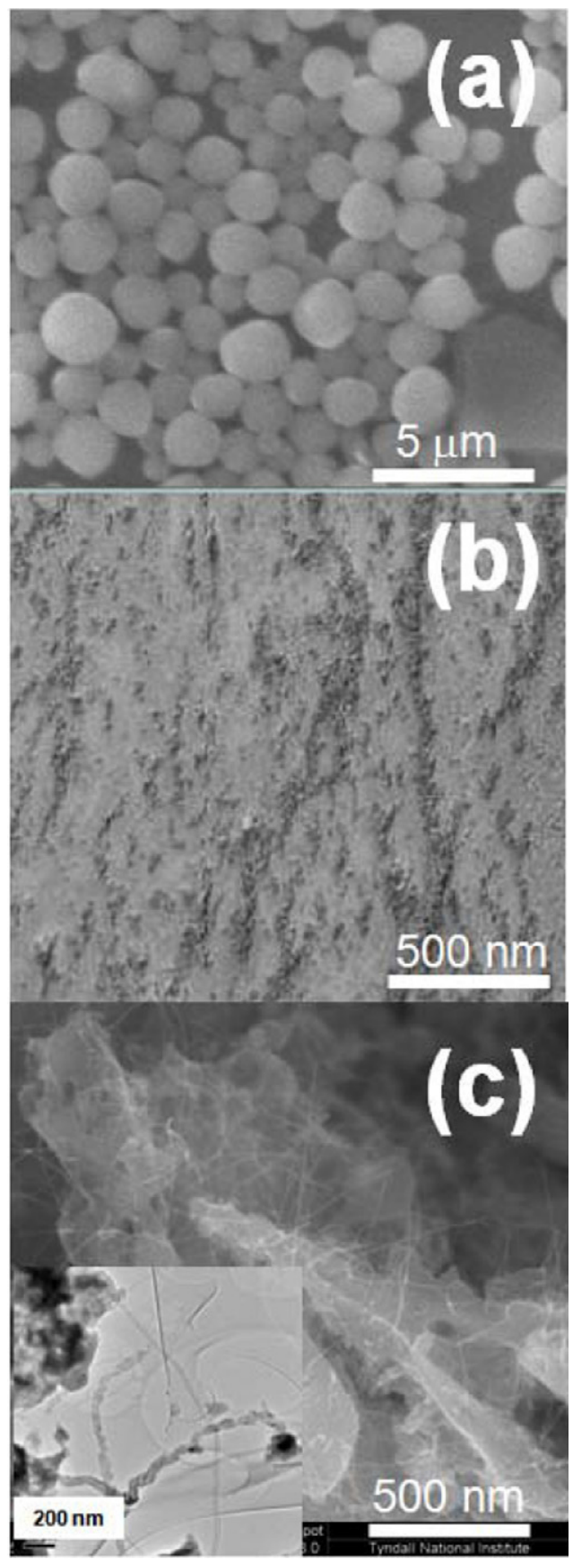

Figure 1. SEM images of Si-ncs after plasma treatment for (a) Si-ncs produced by laser ablation in water (LA-Si-ncs), (b) Si-ncs produced by electrochemical etching (EE-Si-ncs) and (c) EE-Si-ncs fragmented by laser processing in water (LF-Si-ncs). Inset of $(c)$ shows detailed TEM image of the filamentary structures.

main differences for non-metallic compared with metallic catalysts. Firstly, due to the reduced catalytic activity of non-metals, carbon precursors need to reach the surface of the nanoparticles largely decomposed either through higher processing temperatures or through plasma-induced decomposition. Also, it has been shown that the change of Gibbs free energy is more favourable to nucleate and form the graphene cap in a plasma environment at lower temperatures [16]. Secondly, because a molten layer would be difficult to produce on the non-metallic catalyst nanoparticles, carbon
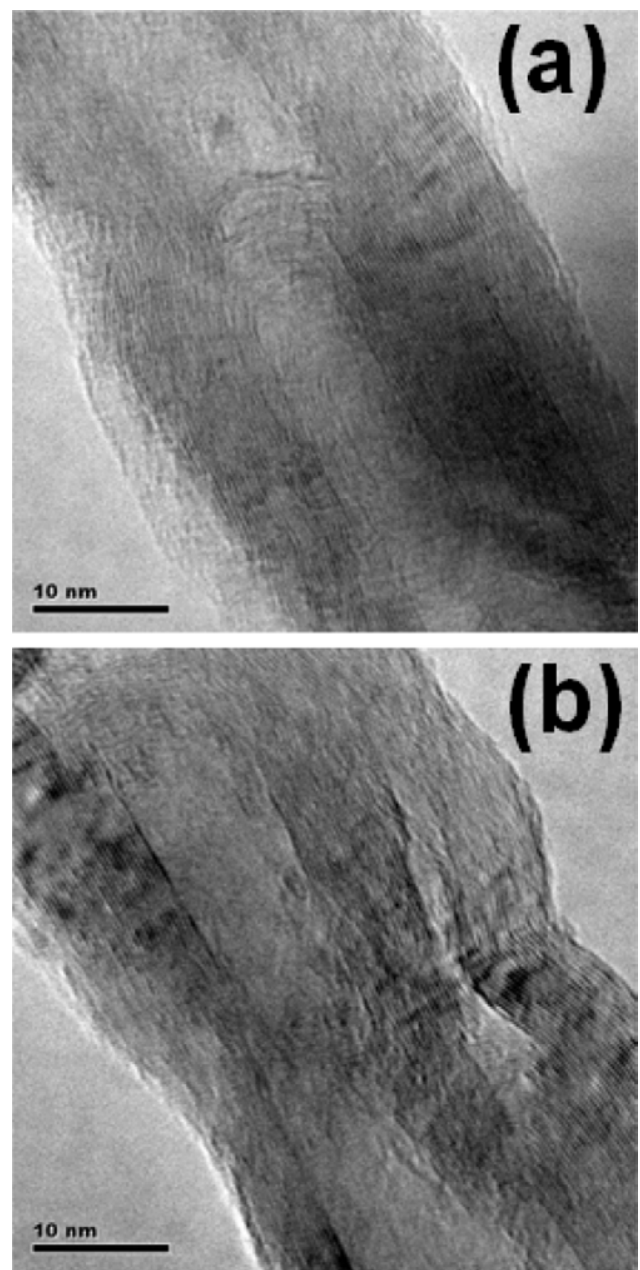

Figure 2. Typical TEM images of MWCNTs grown from samples of Si-ncs that were produced by electrochemical etching and subsequently laser fragmented in water. CNTs were grown without any metal catalyst by exposing the $\mathrm{Si}$-ncs to a microwave plasma-enhanced chemical vapour deposition process.

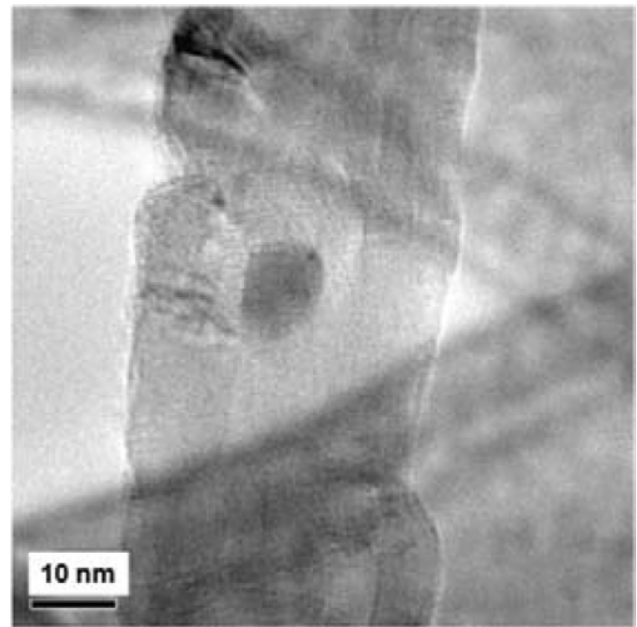

Figure 3. TEM image showing the presence of Si-nc within a carbon nanotube.

atoms can only adsorb and diffuse on the solid surface, i.e. VSS or VSSS. This was also shown via quantum mechanicalmolecular dynamic simulations on $\mathrm{SiO}_{2}$ which confirms that the absence of a molten phase as in the VLS growth [23]. 
Exposition of a silicon surface to a carbon-containing plasmaactivated gas generally leads to the formation of a siliconcarbide layer [24, 25]; however, once a layer of silicon carbide has been formed the carbon diffusion rate into the siliconcarbide layer is low and carbon surface diffusion may occur, consistent with the VSS mechanism. This point is important as the stabilization of a carbon-saturated silicon surface is generally considered a prerequisite for CNT growth. The formation of the $\mathrm{SiC}$ layer has been found to be independent of the oxidation state of the silicon surface; however surface defects have played a role on what happens following the formation of silicon carbide [24]. CNT growth on $\mathrm{SiO}_{2}$ nanoparticles has been studied in some detail [12,14,23] and because our Si-ncs have oxidized surfaces we believe that oxygen has played an important role, possibly by increasing the capture of $\mathrm{CH}_{x}$ radicals. Simulations have in fact shown that simultaneous hydrogen abstraction and formation of $\mathrm{CO}$ gaseous species is a prerequisite for CNT nucleation, which becomes highly favourable via the production of carbon dangling bonds [23]. However, our results also indicate that a high-quality oxide such as in LA-Si-ncs has prevented CNT growth (figure $1(a)$ ) and therefore it may imply that some form of surface defects or dislocations is required to allow for the unstable physisorption of carbon atoms or for the catalytic decomposition of the hydrocarbons $[12,14]$. This may be corroborated by previous works where CNTs could be grown on scratched areas of $\mathrm{SiO}_{2}$ [12], silicon [16] or with $\mathrm{SiO}_{2}$ nanoparticles that may have been characterized by a low-density oxide due to the chemical synthesis route used [14]. According to this scenario, the EE-Si-ncs would have presented suitable surface characteristics; however, the presence of large Si-nc aggregates may have not exhibited the suitable sizes and morphologies to serve as template for the formation of CNT caps. Therefore, LF-Si-ncs with strained oxide bonds and a sufficient number of non-aggregated Sincs have been the only Si-ncs type that allowed an observable growth of CNTs. Although this is a possible scenario based on our initial observations, further work will be required to fully clarify the specific surface chemistry involved in the nucleation of CNTs on Si-ncs.

We have demonstrated that three different Si-nc processing techniques and subsequent exposure to the same low-pressure plasma process result in very different outcomes. Particularly, the results showed that diverse Si-nc surface features are a key factor to determine the growth of CNTs. In particular, Si-ncs with a low-density surface oxide have shown to have better properties to induce CNT growth. A sufficient fragmentation of Si-ncs aggregates into smaller assemblies or even single Si-ncs, which has also resulted to be an important factor.

While it is evident that CNT growth has been induced by the presence of Si-ncs, at this time we are unable to provide full details on the growth mechanisms and on the $\mathrm{Si}$ ncs/CNT interface. However, these initial results indicate a promising research direction that could lead to the fabrication of an ideal and fundamental nanoscale junction between two nanostructures with unique quantum-confinement effects. The possibility of synthesizing Si-ncs/CNT nano-devices is an exciting scientific opportunity with a wide range of application. Future work will be focused in improving the CNT growth yield and in elucidating the growth mechanism, including a full understanding of the conformation of the Si-ncs/CNT junction.

\section{Experimental section}

Si-ncs prepared by laser ablation in water (LA-Si-ncs). Synthesis of Si-ncs by laser ablation used a nanosecond pulsed excimer laser $(\mathrm{KrF}, 245 \mathrm{~nm}, 20 \mathrm{~Hz})$ [26]. A silicon wafer (ptype boron doped, $\langle 100\rangle)$ is placed at the bottom of a glass container and immersed in $10 \mathrm{ml}$ of water. The laser beam is focused onto a $1.5 \mathrm{~mm}$ diameter spot on the wafer surface by a lens [26]. This type of Si-ncs was then stored in the same water that was used during laser processing.

Si-ncs prepared by electrochemical etching (EE-Si-ncs). Powder containing single Si-ncs and Si-ncs aggregates were produced by electrochemical etching [27]. The technique involves the etching of a silicon wafer (p-type boron doped, $\langle 100\rangle, 0.1 \Omega \mathrm{cm}$, thickness $0.525 \mathrm{~mm}$ ) for $1 \mathrm{~h}$ at $1.6 \mathrm{~mA} \mathrm{~cm}^{-2}$ constant current in a HF: ethanol electrolyte (1:4). After the etching process, the resulting porous silicon wafer has been mechanically pulverized [27]. The full process produces a powder of nano-sized crystals and their aggregates [28]. The Si-nc powder was stored in water; however, this type of Si-ncs exhibit a hydrophobic character and therefore a few drops of ethanol have been used to wet the surface of the Sincs/aggregates and allow the powder dispersion in water.

Si-ncs treated by laser fragmentation (LF-Si-ncs). In order to obtain the third type of Si-ncs, $5 \mathrm{mg}$ of the Si-nc powder produced by electrochemical etching (see description above) was dispersed in $20 \mathrm{ml}$ of pure water before being subjected to the laser fragmentation process. Fragmentation by nanosecond pulsed laser requires the $\mathrm{Si}$-ncs to be dispersed in the colloid [28,29], therefore a small amount of ethanol (20 drops) was used to wet the $\mathrm{Si}-\mathrm{nc} / \mathrm{Si}$-nc aggregate surface prior to the introduction into water. The colour of the colloid homogeneously dispersed is yellow. Fragmentation was achieved by irradiating the colloid with a pulsed laser (Kr: F, $245 \mathrm{~nm}, 20 \mathrm{~Hz}, 10 \mathrm{~ns}$ ) at $1.94 \mathrm{~W}$ for $1 \mathrm{~h}$ and at room temperature. The laser beam was focused onto a $3 \mathrm{~mm}$ diameter spot on the liquid surface by a lens with a focal length of $250 \mathrm{~mm}$. During the irradiation, the glass container was closed and rotated. When the nanosecond pulsed laser irradiation is applied, fragmentation of the larger aggregates occurs and at prolonged laser irradiation the solution becomes transparent.

Low-pressure plasma processing of the Si-ncs. All three different types of Si-ncs were stored in water for $\sim 4$ weeks before plasma processing. Before the plasma treatment, the three types of Si-nc colloids were drop-cast on a silicon substrate and left to dry under ambient conditions and therefore exposed to air. The samples were then processed using a microwave plasma-enhanced chemical vapour deposition (MPECVD) technique [30]. We have pre-treated the substrate 
using $\mathrm{N}_{2}$ plasma at $300 \mathrm{~W}, 20 \mathrm{mbar}$ and $750^{\circ} \mathrm{C}$ for $3 \mathrm{~min}$. After pre-treatment, an argon/methane mixture $(30 \mathrm{sccm}$ and $10 \mathrm{sccm}$ respectively) was introduced and the power was reduced at $600 \mathrm{~W}$; processing continued for a further $5 \mathrm{~min}$.

Material characterization. Fourier transform infra-red spectroscopy (FTIR) was carried out with a Perkin-Elmer Spectrum 2000; the colloids were drop-cast and dried on a silicon wafer. FTIR measurements were taken in ratio mode using as reference a section from the same silicon wafer where the Si-ncs were deposited. The range $370-7800 \mathrm{~cm}^{-1}$ was scanned with a resolution of $1 \mathrm{~cm}^{-1}$ and $0.2 \mathrm{~cm}^{-1}$ steps. Photoluminescence (PL) of the Si-ncs was carried out with a He : Cd laser $(325 \mathrm{~nm})$. The excitation laser intensity was approximately $\sim 70 \mathrm{~mW} \mathrm{~cm}^{-2}$. SEM and TEM images were taken with a Hitachi S-4300 microscope at $20 \mathrm{kV}$ and a JEOL JEM-22011 with a LaB6 filament at $200 \mathrm{kV}$ acceleration voltages, respectively. Energy-dispersive $\mathrm{x}$-ray spectroscopy was employed to determine local elemental concentrations.

\section{Acknowledgments}

This work was partially supported by a NEDO project. DM thanks the support of the JSPS Invitation Fellowship (Japan).

\section{References}

[1] Grom G et al 2000 Nature 407 358-61

[2] Martel R et al 2001 Phys. Rev. Lett. 87256805

[3] Mangolini L, Thimsen E and Kortshagen U 2005 Nano Lett. 5 655-9

[4] Avouris P and Dimitrakopoulos C 2012 Mater. Today 15 86-97

[5] Ibrahim I, Bachmatiuk A, Warner J H, Büchner B, Cuniberti G and Rümmeli M H 2012 Small 8 1973-92

[6] Sankaran R M 2011 J. Phys. D: Appl. Phys. 44174005

[7] Lee S W, Mattevi C, Chhowalla M and Sankaran R M 2012 J. Phys. Chem. Lett. 3 772-7
[8] Chiang W-H and Sankaran R M 2009 Nature Mater. 8 882-6

[9] Ostrikov K and Mehdipour H 2012 J. Am. Chem. Soc. 134 4303-12

[10] Chiang W-H and Sankaran R M 2012 Carbon 50 1044-50

[11] Sankaran R M 2011 J. Phys. D: Appl. Phys. 44174005

[12] Hong G, Chen Y, Li P and Zhang J 2012 Carbon 50 2067-82

[13] Švrček V, Ersen O, Dintzer T, Le Normand F, Pham-Huu C and Ledoux M J 2006 Appl. Phys. A 83 153-7

[14] Chen Y and Zhang J 2011 Carbon 49 3316-24

[15] Gohier A, Laik B, Kim K-H, Maurice J-L, Pereira-Ramos J-P, Cojocaru C S and Van P T 2012 Adv. Mater. 24 2592-7

[16] Kumar S, Mehdipour H and Ostrikov K 2013 Adv. Mater. 25 69-74

[17] Levchenko I, Ostrikov K, Mariotti D and Švrček V 2009 Carbon 47 2379-90

[18] Švrček V, Mariotti D, Shibata Y and Kondo M 2010 J. Phys. D: Appl. Phys. 43415402

[19] Okamoto C, Tsujiya K, Minemoto T, Murozono M, Takakura H and Hamakawa Y 2005 Japan. J. Appl. Phys. 44 8351-5

[20] Wang D et al 2011 Adv. Funct. Mater. 21 4010-8

[21] Zhou S, Liu X and Wang D 2010 Nano Lett. 10 860-3

[22] Švrček V, Mariotti D, Kalia K, Dickinson C and Kondo M 2011 J. Phys. Chem. C 115 6235-42

[23] Page A J, Chandrakumar K R S, Irle S and Morokuma K 2011 J. Am. Chem. Soc. 133 621-8

[24] Demuynck L, Arnault J C, Polini R and Le Normand F 1997 Surf. Sci. 377 871-5

[25] Zhu W, Wang X H, Stoner B R, Ma G H M, Kong H S, Braun M W H and Glass J T 1993 Phys. Rev. B 47 6529-42

[26] Švrček V, Mariotti D and Kondo M 2009 Opt. Express 17 520-7

[27] Švrček V, Slaoui A and Muller J C 2004 J. Appl. Phys. 95 3158-63

[28] Švrček V, Sasaki T, Shimizu Y and Koshizaki N 2006 Chem. Phys. Lett. 429 483-7

[29] Švrček V, Mariotti D, Kalia K and Kondo M 2009 Chem. Phys. Lett. 478 224-9

[30] Mathur A, Roy S S, Tweedie M, Maguire P D and Mclaughlin J A 2009 J. Nanosci. Nanotechnol. 9 4392-6 